\title{
Transaction
}

\section{A Novel Random Preferential Orientation of the Crystal A-axis Along the Radial Direction Confirmed on the Poly-p-phenylenebenzobisoxazole (PBO) Fiber Made with a Water Vapor Coagulation Method}

\author{
Tooru Kitagawa", Kohei Kiriyama, and Yusuke Shimizu \\ Membrane-structural Development Group, Corporate Research Center, Toyobo Co., Ltd. \\ 2-1-1 Katata, Otsu, Shiga 520-0292, Japan
}

\begin{abstract}
This article concerns PBO fiber structure, especially a preferential orientation of the a-axis of the PBO crystal in the fiber. It has been reported that the a-axis of the PBO crystal aligns parallel to the radial direction on the round cross section for PBO AS and HM fibers. This observation was proved by selected-area electron diffraction and micro-focus X-ray diffraction. An application of water vapor coagulation is carried out in this study. It is proved that the fiber so made shows a random orientation of the PBO crystal a-axis on the cross section. This fact has never been reported on any scientific or engineering journals before as far as the authors have concerned and it becomes state-ofthe-art in this study. A mechanism to explain the presence and/or absence of such preference is proposed. The authors believe that the presence of this radial preferential orientation comes from a combination or a balance of polymersolvent interaction and the direction of diffusion for solvent from the center to the outside of the fiber.
\end{abstract}

(Received 9 February, 2015 ; Accepted 18 May, 2015)

\section{INTRODUCTION}

PBO (poly- $p$-phenylene benzobisoxazole) is a molecule that has a rigid-rod backbone made from phenylene and oxazole rings. It is formed from a complex ring but the direction of the molecule is close to a straight line ${ }^{1,2}$. Therefore, the choice of a good solvent for the molecule is much limited for spinning and polyphosphoric acid is a reasonable choice for selecting a good solvent ${ }^{3}$. The fiber can be spun from dope (concentrated solution) made from $\mathrm{PBO}$ molecules and poly-phosphoric acid, then coagulated, washed, dried and heat-treated if necessary. Because the PBO molecule take a relatively straight conformation in the fiber, the molecular orientation is proved to be very high along the fiber axis, whereas the interaction between molecules normal to the fiber direction is not so strong ${ }^{4-17}$. Therefore, the fiber shows relatively small crystallite size, especially along the a- and b-axes. Here, the diffraction spots are indexed after Fratini et al. ${ }^{18}$ in this article.

One of interesting points to be taken into consideration is the presence of a preferential orientation of the crystal a-axis on the cross section of PBO fibers ${ }^{12,19}$. It was shown that the a-axis aligns in a radial manner by

\# corresponding author

E-mail :Tooru_Kitagawa@toyobo.jp analyzing a microtomed section of the fiber together with electron diffraction (ED) focusing a small aperture on a certain area with interest and tilting the sample ${ }^{12}$. The principal way of its analysis is as follows. Because the fiber keeps a preferential orientation in a microtomed specimen, the direction of the a-axis of the PBO crystal aligns almost parallel to the radial direction on a round cross section of the fiber. For the specimen the diffracted ED pattern taken from the surface area gives relatively much intensity of the (200) diffraction together with almost less intensity from the (010) and (210) diffractions, whereas the intensity of the (010) and (210) diffractions become slightly much from the center part of the section. As for poly- $p$-phenyleneterephthalamide (PPTA) the fiber shows a same kind of preferential orientation for the crystal b-axis and the fact was reported by Yabuki et $\mathrm{al}^{20}$ by analyzing an interference pattern with a polarization microscope from an inclined cross section of a sample fiber. Same kind of analysis for analyzing this preferential orientation for PPTA also has been carried out with a micro-focus X-ray diffraction method at the ID 13 beam line of the European Synchrotron Facility (ESRF) ${ }^{21}$. This beam line also reported the presence of the radial preferential orientation for the $\mathrm{PBO} \mathrm{AS}, \mathrm{HM}$ and $\mathrm{HM}+$ fibers ${ }^{19,22}$. The $\mathrm{PBO} \mathrm{HM}+$ fiber is made through nonaqueous coagulation with heat-treatment and the PBO AS is a standard type made through aqueous coagulation and 
the PBO HM is a high-modulus type made through heattreatment of the AS fiber. However, no one has given a reasonable reason why the three fibers show such radial preferential orientation for a certain crystal axis up to now.

This time the authors encounter a novel idea to make fiber coagulated with water vapor. The state-of-the-art in this study lies in the point in which the process of changing coagulant from a liquid solvent to water vapor gives a totally different preferential crystal orientation of PBO. In the PBO solution before spinning, the molecule is so rigid that it forms liquid crystal but the direction of the liquid crystal should be random, because there is no specific physical force applied to the anisotropic solution. The fiber filament that is just extruded from the spinneret is a very thin thread that is formed from a drawn concentrated mixture of PBO and poly-phosphoric acid. This thread contains the ordered region that forms liquid crystal but the direction shouldn't orient to any specific preferential direction except to the drawing direction. Strictly speaking the direction of the ordered domain is under no constraint and should be random as a result except for the direction to the drawing direction.

After passing through an air gap the filament is coagulated and solidification occurs. In the coagulation process the filament made from poly-phosphoric acid and PBO molecule is soaked in a relatively massive aqueous solution. The diffusion of the solvent from the inside and the non-solvent from the outside occurs simultaneously and mutually in the fiber. From the outside a water molecule as a coagulant dissolves in the filament made from concentrated $\mathrm{PBO}$ solution, whereas polyphosphoric acid as a good solvent for PBO molecule comes off the solute molecule and diffuses to the outside of the fiber. Then the PBO molecule can't take a liquidcrystalline form any longer, because the poly-phosphoric acid surrounded the PBO molecule no more exists and the solute (PBO molecule) may be surrounded by coagulant. Therefore, the liquid crystalline domains transform to a solid form with including crystallites.

Here, the authors will confess the most important point. If coagulation is made with a one-way manner more rapidly, a characteristic coagulation process is allowed without the outflow of the solvent induced by adopting liquid coagulant. The resulting fiber may not have a certain preferential orientation already reported. There may give a less tendency to show a radial pattern, because the very short period of time for structure formation may allow the fiber to keep the inclusion of original structure before coagulation. Therefore, they tried a coagulation method with water vapor to make a new type of PBO fiber and the fiber structure so made is analyzed. Because water vapor is applied under relatively high temperature, its water molecule goes through inside the fiber and reaches the center in a very short time with a one-way manner, so coagulation without the outflow of the solvent followed by a fixation of structure will be carried out. This procedure may keep an original structure as liquid crystal in the coagulated fiber.

\section{EXPERIMENTAL}

\section{Fiber making}

PBO fibers were produced with a dry-jet wet spinning method ${ }^{23}$. A PBO polymer, synthesized and polymerized according to the literature ${ }^{3}$, was dissolved in a solvent (poly-phosphoric acid) and was supplied to a spinneret from a solution tank (reservoir). The fiber extruded from the spinneret was led to a coagulation bath filled with an aqueous solution as a coagulant generally in the normal case. In this study hot water having a temperature of $85^{\circ} \mathrm{C}$ was adopted as a coagulant for a comparative case. The coagulated PBO fiber was successively subjected to washing, drying, and wound on a winder. To raise its fiber modulus it was heat-treated further.

In the case for water vapor coagulation an ejector is adopted to apply water vapor at high temperature (steam) to the filaments made from a PBO concentrated solution just after they were running through the air gap before touching a coagulant, as shown in Figure 1. The ejector is designed as the way water vapor is applied homogeneously to the filaments and it makes a steady flow so as not to disturb the running of the filaments. The time duration of the running filaments passing through the ejector was less than one second and the temperature

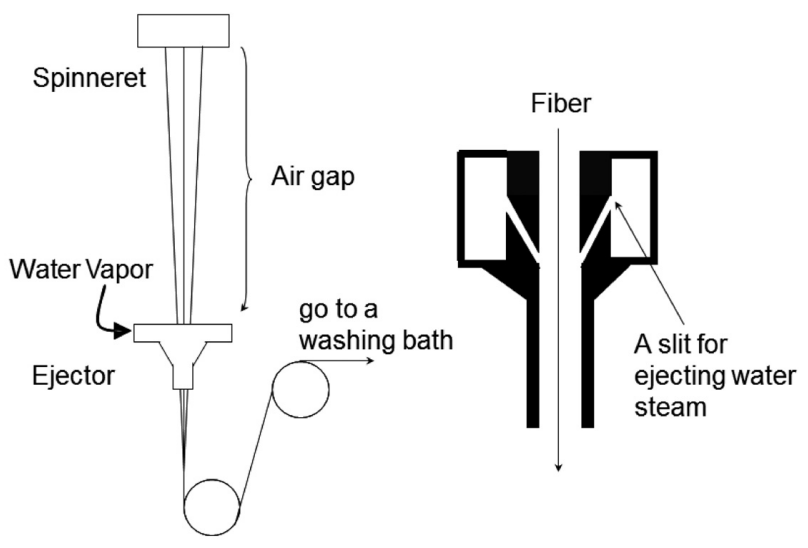

Fig. 1 Schematic diagram for water vapor coagulation (left) and an ejector (right). 
Table 1 Mechanical properties for heat-treated PBO fibers investigated in this study.

\begin{tabular}{cccc} 
Coagulation & $\begin{array}{c}\text { Strength } \\
\mathrm{GPa}\end{array}$ & $\begin{array}{c}\text { Modulus } \\
\mathrm{GPa}\end{array}$ & $\begin{array}{c}\text { fineness } \\
\text { dtex }\end{array}$ \\
\hline Water Vapor & 4.2 & 248 & 1.0 \\
Hot Water & 4.7 & 279 & 1.0
\end{tabular}

inside the ejector was controlled at $97^{\circ} \mathrm{C}$. After passing through the ejector the filaments were guided to a washing process with water, a drying oven and a winder as the same with the normal process. Then the fiber was heat-treated under tension. The tensile properties for both the fibers are tabulated in Table 1.

\section{Wide-angle $X$-ray Scattering}

An X-ray generator on a diffractometer (Rigaku RINT-2500PC rotating anode type) was operated at a power of $40 \mathrm{kV} \times 200 \mathrm{~mA}$. Nickel-filtered $\mathrm{CuK}_{\alpha}$ beam was used as an X-ray source. The pinhole collimator of 0.15 $\mathrm{mm}$ in diameter was used. A yarn (166 filaments) of 500 $\mathrm{cm}$ in length was wound on a sample holder, and then it was set on a goniometer system with a pulse-height analyzed proportional counter. Wide-angle X-ray diffraction intensities on the equator and on the meridian were recorded with the symmetry-transmission method. The details of data analysis and correction for beam broadening are reported in the literature ${ }^{12}$.

\section{Electron Microscopy and Electron Diffraction}

A commercially available epoxy resin (a mixture of Nakarai Ruabekku 812, Epicoat 812 and Ruabekku DMP30) was adopted to embed fibers, which was kept in an oven at $60^{\circ} \mathrm{C}$ for 24 hours for curing. Firstly, the resin block was cut and polished with a glass knife to form a suitable shape in which a single fiber was located on the tip of a polygonal pillar in order to get an ultrathin section with a microtome (Reichert-Nissei Ultracut N) and a diamond knife (Sumitomo Sumiknife). Then the fiber was microtomed along the fiber parallel to the fiber axis. The number of sections microtomed was up to about 120 in total from the beginning to the end to recover the whole part of the fiber over the diameter and all the sections microtomed were collected on a carbon-coated thin film. Then it was put on a sample holder for TEM (transmission electron microscope) observation and a selected-area electron diffraction (SAED) pattern was taken adopting a selected area aperture to see the structural difference between the surface and core parts. An electron microscope (JEOL JEM-2100) was operated at an accelerated voltage of $200 \mathrm{kV}$ with an aperture, the diameter of which was seen about one micrometer on the sample position, and SAED patterns were recorded on a CCD camera (Gatan Orius SC 1000). The schematic diagram to microtome a fiber and a bright-field image that indicates the position of the selected area by an aperture on an ultrathin section for electron diffraction is illustrated in Figure 2.

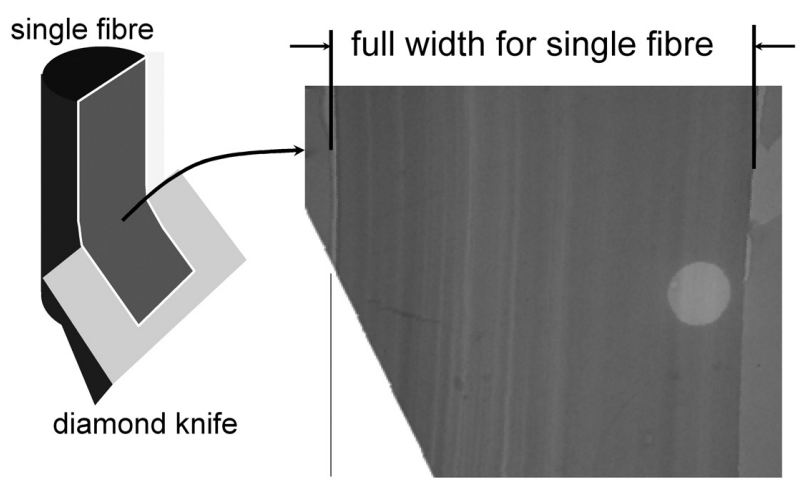

Fig. 2 Schematic diagram for microtoming a section with a diamond knife. A bright field image for a section is shown with a mark of an aperture.

\section{Micro-Focus $X$-ray diffraction}

Micro-focus X-ray diffraction patterns were taken at the European Synchrotron Radiation Facility (ESRF) on the beam line ID13 (micro-focus beam line) ${ }^{19,24}$. The experimental identification number was IN-617 on September 3, 2007. The diameter of the micro-focus Xray beam was adjusted to approximately one micrometer and the wavelength for measurement was $0.0961 \mathrm{~nm}$. A single fiber was mounted on a sample bed which can move horizontally along the direction normal to the beam keeping high spatial resolution. Then the fiber was moved forward with a stepwise manner to the beam, a shot of Xray diffraction was taken and it was moved forward again to the next position for measurement. This data collection procedure continued until the fiber got across the beam up to the poison in which the beam became out-of focus completely. The diffraction data were registered on a MarCCD two-dimensional detector.

\section{RESULTS AND DISCUSSION}

\section{Apparent Crystallite Size}

Figure 3 shows the X-ray diffraction patterns on the equator and on the meridian for PBO fibers made through a water vapor coagulation process with and without heattreatment under tension after sample fibers passing through a draying process. It is understood that these patterns are principally same except that there is a difference in sharpness of peaks between the two fibers. If they are compared with the patterns of the AS, $\mathrm{HM}^{12}$ and 

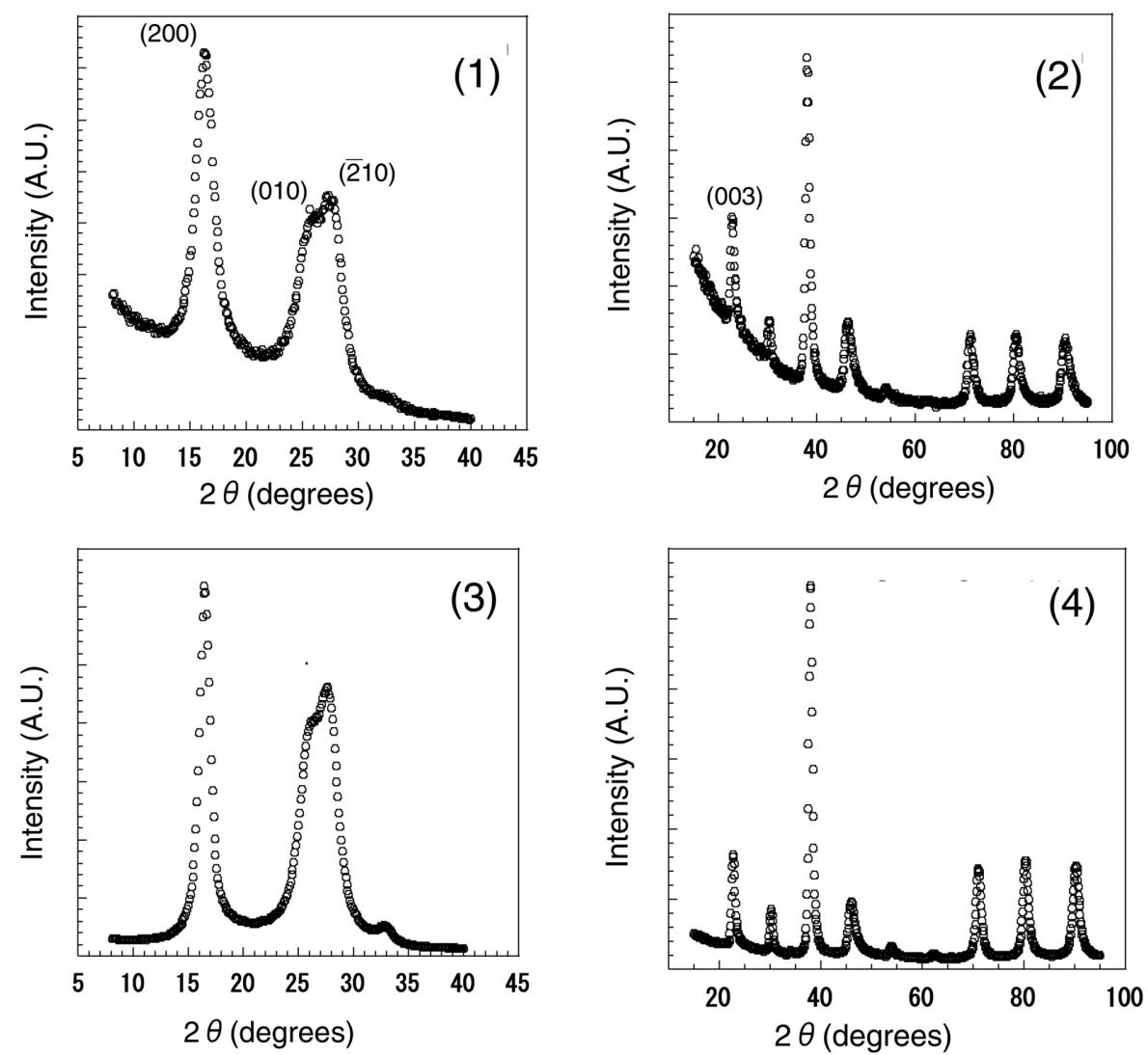

Fig. 3 Wide-angle X-ray diffraction patterns for water vapor coagulation fibers with/without heat-treatment. (1) Equatorial scan without heat-treatment, (2) Meridional scan without heat-treatment, (3) Equatorial scan with heat-treatment, (4) Meridional scan with heat-treatment.

$\mathrm{HM}+{ }^{13}$ PBO fibers, they are explained as the same way in that the pattern is same but the sharpness of each spot is different. These facts suggest that the crystal structure produced with water vapor coagulation attributes to the same crystallographic system proposed by Fratini et al. ${ }^{18}$ but their crystallite size and orientation are different. By approximating the profile of diffraction peaks with a combination of Gaussian and Lorentzian functions, the values of full width at half height maximum are estimated for the profiles for the (200), (010) and (210) diffractions. Apparent crystallite size is calculated with Scherrer's equation and the result is tabulated in Table. 2.

Table 2 Apparent crystallite size for fibers made through a water vapor coagulation process. The values for the $\mathrm{PBO} \mathrm{HM}$ and $\mathrm{HM}+$ fibers are also shown ${ }^{12,13}$.

$\begin{array}{cccc}\text { Index } & (200) & (010) & (003) \\ \text { Fiber } & \mathrm{nm} & \mathrm{nm} & \mathrm{nm}\end{array}$

Water Vapor Coagulation

$\begin{array}{lrrr}\text { Drying } & 5.2 & 3.7 & 12.3 \\ \text { Heat-Treatment } & 8.1 & 3.5 & 12.6 \\ \mathrm{HM} & 11.0 & 4.2 & 16.0 \\ \mathrm{HM}+ & 11.0 & 4.6 & 16.2\end{array}$

It is understood that the values of apparent crystallite size for the (200) and (003) diffractions become large with heat-treatment for water vapor coagulation. However if the values are compared with the ones for the PBO HM and $\mathrm{HM}+$ fibers, they are less. The water vapor coagulation gives relatively small crystallite size in the fiber even if the fiber is produced with heart-treatment.

Comparison between the surface and core regions with Selected Area Electron Diffraction

Figure 4(1) shows the SAED patterns for water vapor coagulation from the surface and core regions. We can find there are two strong diffraction spots from the (200) and a combination of the (010) and $(\overline{210})$ planes on the equator for both two images, indicating that there seems to be less preferential orientation of the a-axis of the PBO crystal in the fiber, because both two diffraction spots from different crystal planes keep strong intensity irrespective of the electron beam position for the surface and core regions illuminated on a ultrathin section.

In Figure 4(2), the SAED patterns from the surface and core regions for the fiber made with hot water $\left(85^{\circ} \mathrm{C}\right)$ coagulation followed by heat-treatment are shown. The intensity due to a combination of the (010) and $(\overline{2} 10)$ is relatively weak to that of the (200) plane from the surface 

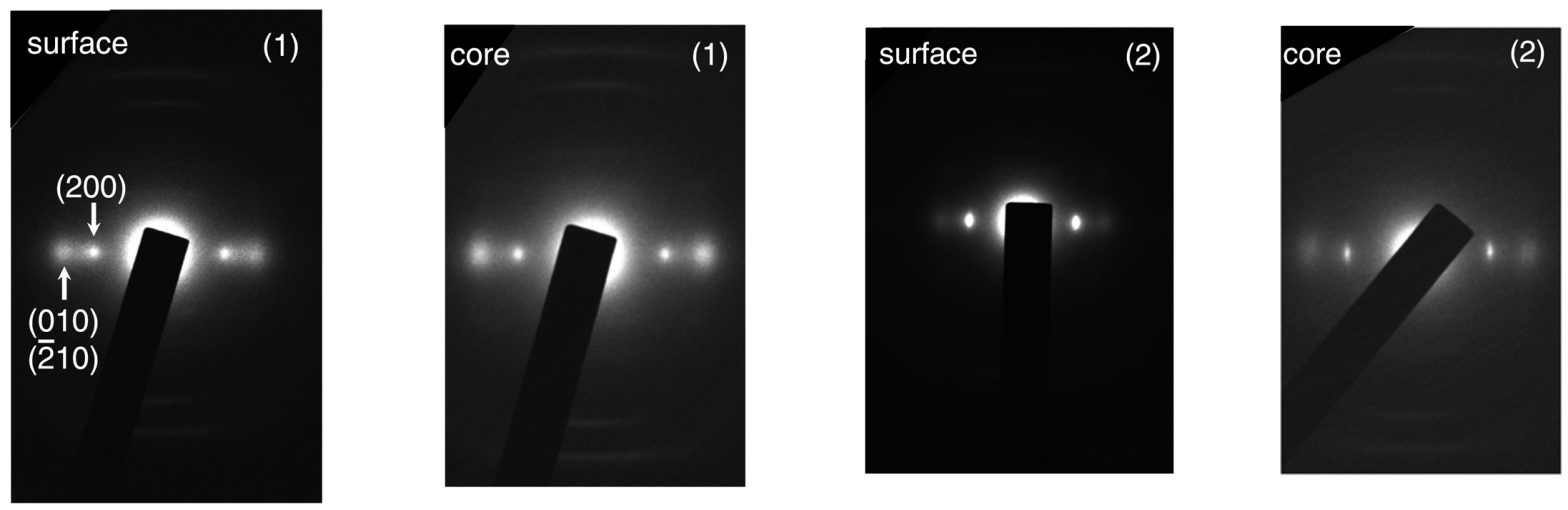

Fig. 4 SAED patterns for the heat-treated fibers (1) with water vapor coagulation and (2) hot water coagulation. The diffraction patterns are taken for the surface and core regions on an ultrathin section.

region, but the intensity of the combined spots becomes relatively much from the core region, suggesting there seems to be preferential orientation in the fiber structure, which is similar to the case that has been already shown for the PBO AS and HM fibers ${ }^{12}$.

Figure 5 shows the equatorial SAED profiles from the surface region of the section. The intensity ratio $\left(S_{B} / S_{A}\right.$ in the figure) of a combination of the (010) and (210) diffractions $\left(S_{B}\right)$ to the $(200)$ diffraction $\left(S_{A}\right)$ from the surface region on an ultrathin section is compared for the fibers made with water vapor coagulation and hot water coagulation. This ratio becomes more in the case of water vapor coagulation than that of hot water coagulation. This tendency also supports the above-mentioned hypothesis that there is less preferential orientation of the a-axis of the PBO crystal in the fiber structure for the fiber made through a water vapor coagulation process than that through a hot water coagulation process.

\section{Diffraction Profile for the (200) of PBO crystal across the fiber}

To estimate the direction of the a-axis of the PBO crystal in the fiber more preciously, a micro-focus X-ray diffraction analysis is adopted. This method has been developed and raised by the ID 13 group at ESRF ${ }^{19,21,24}$. They proposed a model to calculate the diffraction intensity profile from a single fiber as a function of the position at which the fiber is illuminated by the source $\mathrm{X}$ ray. It should be noted here that all the (200) crystal planes that intersect the X-ray beam passing through the fiber can not contribute to the intensity of the (200) diffraction spot. Only the scattered X-ray from the crystal plane that can satisfy Bragg's law can be diffracted and registered on a two-dimensional detector. A theoretical intensity profile is calculated and compared with the measured data later.

Figure 6 shows a X-ray diffraction pattern taken with a micro-focus X-ray beam line, Figure 7 (1) the intensity profile of the (200) diffraction of the PBO crystal as a function of the position at which a micro X-ray beam is focused on the fiber made through water vapor coagulation with heat-treatment, and Figure 7 (2) on the one made through hot water coagulation with heattreatment. Here, the zero position on the horizontal axis corresponds to the center of the fiber. The intensity profile changes as the way of coagulation. The hot water

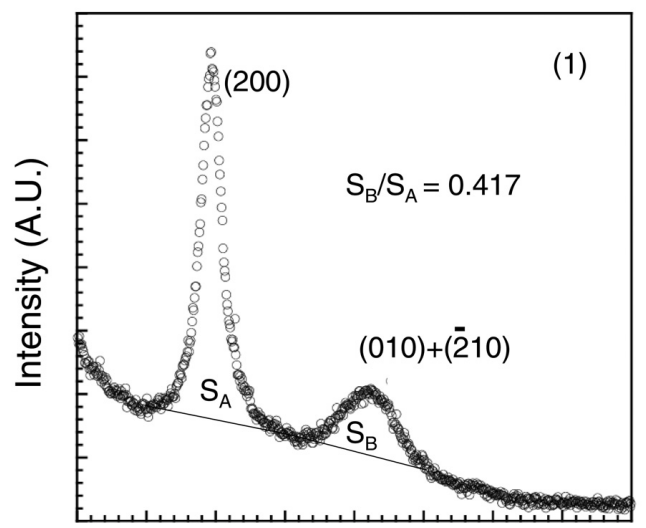

Diffraction Angle (A.U.)

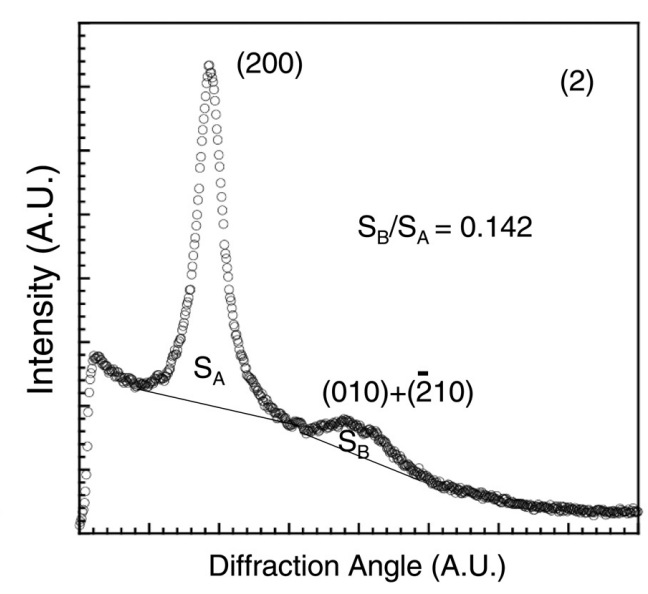

Fig. 5 Equatorial SAED diffraction patterns for heat-treated fibers with (1) water vapor coagulation and (2) hot water coagulation. The selected area aperture was set on the surface region of an ultrathin section. $S_{A}$ stands for the area of the (200) diffraction and $S_{B}$ for the $(010)$ and $(\overline{2} 10)$ diffractions, respectively. 


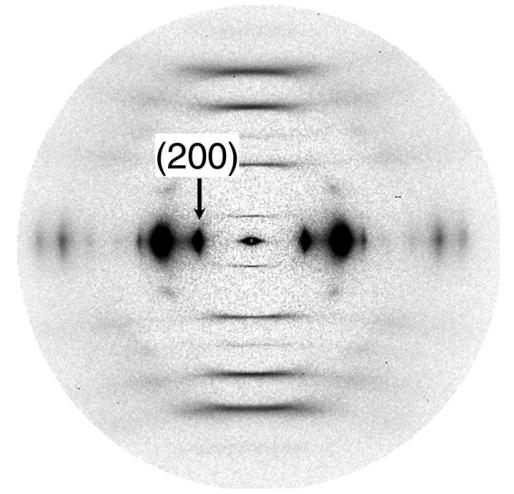

Fig. 6 X-ray diffraction pattern taken with a microfocus X-ray beam.

coagulation case shows a typical pattern having two maxima at off-center positions, indicating the existence of the preferential orientation for the (200) crystal plane as already reported for the PBO AS and HM fibers ${ }^{12,19}$. The water vapor coagulation shows a different pattern against the fiber for the hot water coagulation case and has a single maximum at which the maximum comes where the $\mathrm{X}$-ray beam focuses on the center of the fiber, indicating that the direction of the a-axis of the PBO crystal shows random distribution over all the region of the fiber. This fact has never been reported on any scientific or engineering journals as far as the authors have known.

To prove this existence of random distribution theoretically the intensity profile for the (200) plane of the PBO crystal across the fiber is calculated on the basis of a model according to the articles of Riekel and Davies et $\mathrm{al}^{21,24}$. If the distribution for the (200) crystal plane is random in the fiber, the number of the plane per volume that satisfy Bragg's law is constant at any point of the fiber. Therefore, the intensity is proportional to the scattering volume as the X-ray beam pass through the fiber. The exact formula for the intensity can be expressed

(1) Water vapor coagulation

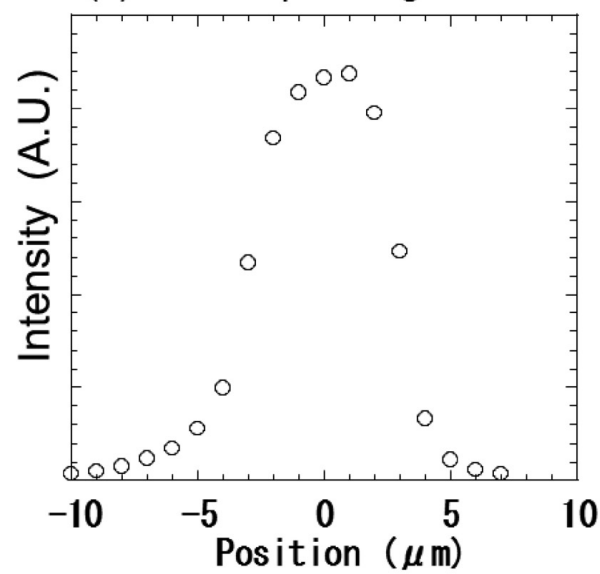

as an analytical function as follows :

$$
\begin{aligned}
& I(r) \propto \int_{r-0.5}^{r+0.5}\left(r_{0}^{2}-x^{2}\right)^{0.5} d x \\
& \propto \frac{\sin (2 b)-\sin (2 a)}{4}+\frac{b-a}{2} \\
& \text { conditions }: r+0.5=r_{0} \sin (b) \\
& r-0.5=r_{0} \sin (a)
\end{aligned}
$$

where $r_{0}(\mu \mathrm{m})$ is the radius of the cross section of the fiber and $r(\mu \mathrm{m})$ the position from the center of the fiber. The range for the integral is set to the width of the micro $\mathrm{X}$ ray beam (1.0 micro meters). The comparison between $\mathrm{X}$ ray intensities measured and the theoretical values obtained with the above equation is performed in Figure 8. Here, the fiber diameter is estimated to be 9.0 micro meters. This value is calculated from the value of measured fineness for a single PBO fiber assuming the value of density for PBO fiber ${ }^{12}$. The measured data points are well fitted with the calculated line, indicating that the direction of the a-axis for the fiber made through a water vapor coagulation process with heat-treatment doesn't take a certain preferential orientation and is concluded as random orientation. No one has reported the presence of this random orientation of the a-axis on the cross section in the fiber structure for PBO fibers with a micro-focus X-ray diffraction method in the literature before this study is present. Further structural analyses along the fiber $\operatorname{axis}^{25}$ and on the quantification of the preferential orientation $^{26}$ of the PBO crystal a-axis will be presented in separate publications.

A proposed mechanism why the preferential orientation appears

1. Normal Coagulation with liquid aqueous coagulation for the AS and HM fibers

Firstly, a PBO molecule in a filament made from a

\section{(2) $85^{\circ} \mathrm{C}$ hot water coagulation}

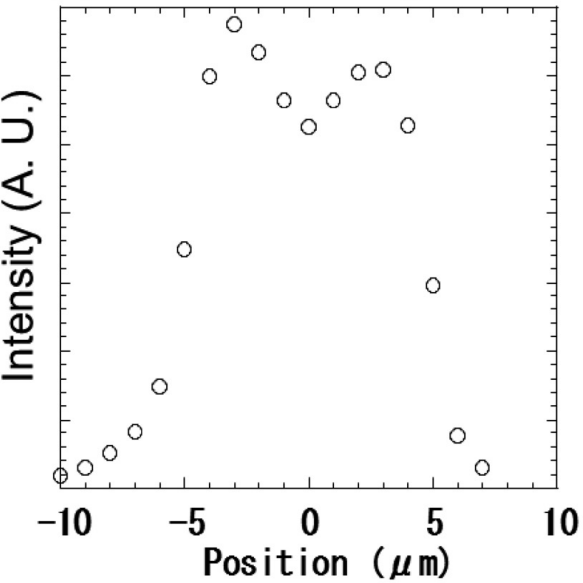

Fig. 7 Intensities of the (200) diffraction against the position of the fiber on which a micro X-ray beam is focused. (1) Water vapor coagulation and heat-treatment, (2) Hot water coagulation and heat-treatment. 


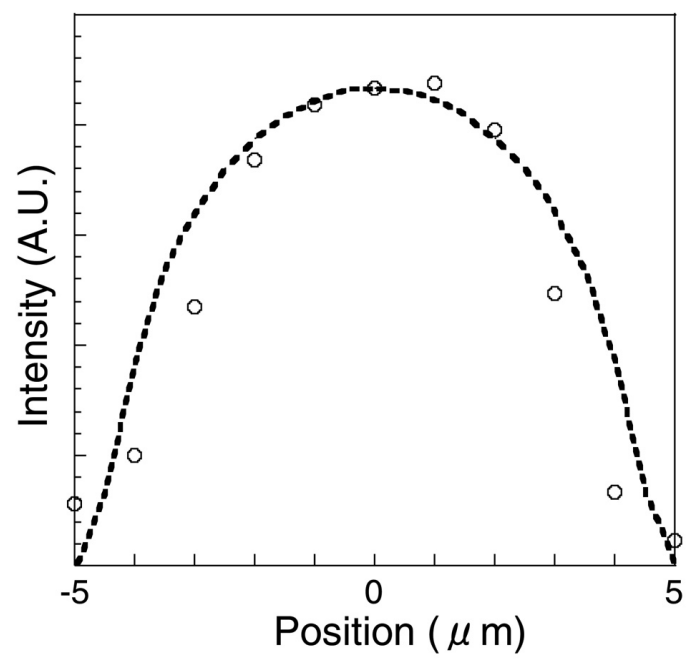

Fig. 8 Comparison between theoretical values (broken lines) and measured data (open circles) for the intensity of the (200) diffraction against the position on which a micro X-ray beam is focused.

concentrated PBO solution passing through an air gap is in the state of an anisotropic solution and surrounded by poly-phosphoric acid. When the dope filament is soaked in a coagulation bath filled with an aqueous solution, a water molecule (coagulant) dissolves into the inner part of the dope filament. The poly-phosphoric acid molecule used as a solvent for $\mathrm{PBO}$ is also soluble to a coagulant and may come off the PBO molecule, because the interaction to the coagulant is stronger than that to PBO. After the detachment from the PBO molecule, the polyphosphoric acid diffuses to the outside of the surface. It is noted that the direction of the flow of the poly-phosphoric acid depicts a radial pattern as a whole on the cross section of the fiber. We propose a hypothesis that in the course of detachment of the phosphoric acid from the PBO molecule and its outflow to the outside, the acid molecule can rotate the phenylene ring in the $\mathrm{PBO}$ molecule and align the phenylene ring of PBO molecule to the direction of the flow (Figure 9(1)), because there is the chemical force of solvation between PBO and polyphosphoric acid. It should be noted that the direction of the phenylene ring normal to the molecular axis is almost parallel to the direction of the a-axis of the PBO crystal ${ }^{18}$, suggesting the reason of presence of the radial pattern of the a-axis in the fiber structure is due to this outflow of the solvent by coagulation.

\section{Water vapor induced coagulation}

The following is a proposed mechanism about what is happening when the filament made from a concentrated PBO solution is coagulated with water vapor in an ejector. The vapor is composed of water molecules with a temperature over $100^{\circ} \mathrm{C}$. The water molecule is thought to be dissolved into the filament in a very shorter period of time than the normal coagulation case. Most part of the fiber is coagulated with water vapor. When a water molecule approaches a poly-phosphoric acid molecule in the filament, because the temperature is high, the polyphosphoric acid may be hydrolyzed to phosphoric acid immediately without its outflow from the original position near $\mathrm{PBO}$ molecule it is attached to. The reaction of hydrolysis is almost finished before the filament goes in a washing process. The phosphoric acid made by hydrolysis with water vapor still stays in the place close to the PBO molecule and does not play a role of good solvent any more even it still surrounds the PBO molecule. There is less chemical interaction between the phosphoric acid and the PBO molecule. When the filament is washed in a washing process, the water molecule washes the phosphoric acid out of the fiber. Because of the less interaction with PBO molecule, the phosphoric acid has less ability to rotate the direction of the phenylene ring in the PBO molecule to the direction of the flow. Therefore the fiber made with water vapor coagulation should keep an original alignment as the state of liquid crystal and shows random orientation of the aaxis on the cross section of the fiber (Figure 9(2)).

\section{CONCLUSIONS}

In this study a preferential orientation of the a-axis of the PBO crystal is discussed for the PBO fiber made with different coagulation methods. It is proposed that the PBO fiber made through water vapor coagulation shows a novel random orientation for the a-axis on the cross section of the fiber as revealed by SAED and micro-focus
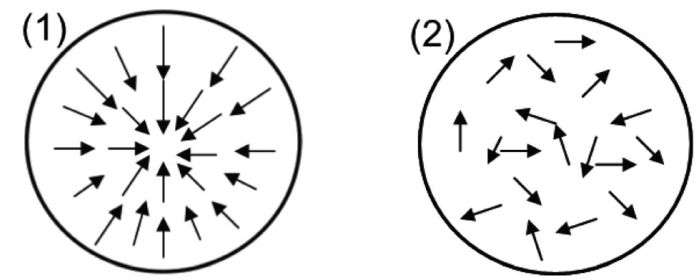

(3)

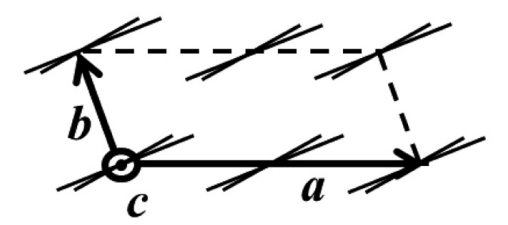

Fig. 9 Schematic drawings to explain the direction of the crystal a-axis of PBO on the cross section of the fiber. (1) radial orientation, (2) random orientation, (3) crystal structure model for PBO proposed by Fratini et $\mathrm{al}^{18}$. 
$\mathrm{X}$-ray diffraction methods. This tendency is different from the fiber made with a liquid coagulation method. The reason why such a different structure presents is discussed. A different mechanism in a coagulation process proceeds and gives a random configuration. Apparent crystallite size for water vapor coagulation shows relatively small values compared with normal coagulation.

One of the authors has involved in elucidating the relationship between fine structures of PBO fibers and their mechanical properties. He has given explanations for many years. This new type fiber with a random preferential orientation of the crystal axis on the cross section may have a different mechanical model, so Raman scattering and X-ray patterns should be measured for the fiber under tension to analyze its inhomogeneity along the fiber axis. Also the change of molecular orientation should be measured under tension to analyze its strain due to the rotation of crystallites. Compressive strength and the behavior of water adsorption as a fiber may be different. It may also be possible for us to get a PPTA fiber having a random crystal axis orientation on the cross section of the fiber. They are all left as a future study.

\section{ACKNOWLEDGEMENTS}

The authors thank Dr. Riekel, Dr. Burghammer and Dr. Davies for their kind help and guidance for microfocus X-ray measurement on the ID 13 beam line at ESRF. Thanks are extended to Mr. Watanuki for preparing fibers, Mr. Funaki for processing twodimensional SAED data, Dr. Murase for microtoming and SAED techniques and Dr. Abe and Mr. Teramoto for valuable discussions. In closing this article one of the authors (T. K.) would like to dedicate these new findings and conclusions to the 70th anniversary of the Society of Fiber Science and Technology, Japan.

\section{REFERENCES AND NOTES}

1. D. B. Roitman and M.McAdon, Macromolecules, 26, 4381 (1993).

2. D. B. Roitman, R. A. Wessling, and J. McAlister, Macromolecules, 26, 5174 (1993).

3. J. M. Wolfe, Encyclopedia of Science and Engineering, Volume 11, Second Edition, 601 (1988).
4. S. J. Krause, T. B. Haddock, D. L. Vezie, P. G. Lenhert, W. F. Hwang, G. E. Price, T. E. Helminiak, J. F. O'Brien and W. W. Adams, Polymer, 291354 (1988).

5. D. C. Martin and E. L. Thomas, Macromolecules, 24, 2450 (1991).

6. S. Kumar, S. Warner, D. T. Grubb and W. W. Adams, Polymer, 35, 5408 (1992).

7. M. E. Hunsaker, G. E. Price and S. J. Bai, Polymer, 33, 2128 (1992).

8. S. J. Bai and G. E. Price, Polymer 33, 2136 (1992).

9. K. Tashiro, J. Yoshino, T. Kitagawa, H. Murase and K. Yabuki, Macromolecules, 31, 5430 (1998).

10. R. J, Young, R. J, Day and M. J. Zakihani, J. Mater. Sci., 25, 127 (1990).

11. S. G. Wierschke, J. R. Shoemaker, P. D. Haaland, R. Pachter and W. W. Adams, Polymer, 33, 3357 (1992).

12. T. Kitagawa, H. Murase and K. Yabuki, J, Polym. Sci. Part B : Polym. Phys., 36, 39 (1988).

13. T. Kitagawa, M. Ishitobi and K. Yabuki, J, Polym. Sci. Part B : Polym. Phys., 38, 1605 (2000).

14. T. Kitagawa, K. Yabuki and R. J. Young, Polymer, 43, 2101 (2001).

15. T. Kitagawa, K. Tashiro and K. Yabuki, J, Polym. Sci. Part B : Polym. Phys., 40, 1269 (2002).

16. T. Kitagawa, K. Tashiro and K. Yabuki, J, Polym. Sci. Part B : Polym. Phys., 40, 1281 (2002).

17. T. Kitagawa, K. Yabuki and R. J. Young, $J$. Macromol. Sci., Phys., B41, 61 (2002).

18. A. V. Fratini, P. G. Lenhert, T. J. Resch and W. W. Adams, Mater. Res. Soc. Sym. Proc., 134, 431 (1989).

19. R. J. Davies, S. J. Eichhorn, C. Riekel and R. J. Young, Polymer, 46, 1935 (2005).

20. K. Yabuki, H. Ito and T. Oda, Sen'i Gakkaishi, 31, T 524 (1975).

21. S. Roth, M. Burghammer, A. Janotta and C. Riekel, Macromolecules, 36, 1585 (2003).

22. T. Kitagawa, H. Sugihara, and M. Tsutsumi, Sen' $i$ Gakkaishi, 71, 105 (2015).

23. H. D. Ledbetter, S. Rosenberg and C. W. Hurting, Mat. Res. Soc. Sym. Proc., 134, 253 (1989).

24. R. J. Davies, M. Burghammer and C. Riekel, Macromolecules, 38, 3364 (2005).

25. T. Kitagawa, J. Macromol. Sci., Phys., in submission.

26. T. Kitagawa, J. Macromol. Sci., Phys., in submission. 